\title{
La dispersion dans les écoulements filaires
}

\author{
par A. Daubert \\ Chef de la Division Recherches \\ Laboratoire National d'Hydraulique, E.D.F., Chatou
}

\section{Réduction des phénomènes à une description filaire}

Lorsque les écoulements se présentent sous la forme d'une veine liquide, c'est-à-dire d'un très long tube de courant, ils appartiennent à la classe des écoulements filaires. C'est en général sous cette forme que se «transfèrent» les grandes masses d'eau sur de longues distances. L'objet de ce chapitre est d'étudier comment ces écoulements transfèrent également ce qui est dissout dans les eaux.

Le grand intérêt porté à ce type d'écoulement a conduit à l'élaboration de théories simplifiées, appelé «modèles», pour en étudier la mécanique plus commodément.

Il est évidemment tentant de compléter ces modèles mécaniques filaires par un modèle cohérent, permettant également de décrire d'une façon simple (c'est-à-dire filaire) les phénomènes de transfert au sein de ces écoulements.

La simplicité du point de vue filaire ne va pas évidemment sans sacrifice. En effet, la contrepartie sera que l'on devra renoncer à connaître la distribution dans une section et se contenter de n'en percevoir que la valeur moyenne.

C'est ainsi que l'on caractérisera le champ de vitesse de ces écoulements par la vitesse moyenne dans chaque section. Cette vitesse «débitante » $U$ n'est de ce fait qu'une fonction de l'abscisse curviligne $x$ de la section et du temps $t$. Les écoulements filaires sont donc des modèles unidimensionnels. Il existe bien d'autres modèles qui sont également unidimensionnels, notamment les couches limites et les jets.

En ce qui concerne les transferts, la seule ambition des modèles filaires sera donc de permettre la détermination de la concentration moyenne $C_{0}(x, t)$ en n'utilisant de la cinématique de l'écoulement que la vitesse débitante $U$. On connaît les difficultés auxquelles se heurte ce genre d'entreprise. Dans la dynamique des écoulements turbulents, cela conduit au fameux tenseur de Reynolds dont on sait qu'il constitue l'obstacle essentiel qui fait que les équations de Reynolds restent pour l'instant lettres mortes.

En effet, considérons, comme à l'habitude, un volume de contrôle limité par deux sections droites de la veine liquide.

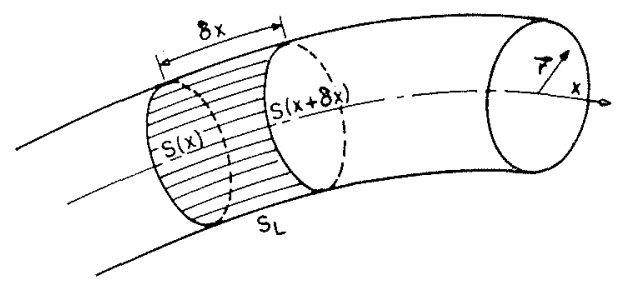

Nous noterons.

$$
\begin{aligned}
& u(\vec{r}, x, t) \text { la vitesse locale } / / \vec{x} \\
& c(\vec{r}, x, t) \text { la concentration locale. }
\end{aligned}
$$

Par définition :

- la vitesse débitante $U(x, t)=\frac{1}{S} \int_{S} u(\vec{r}, x, t) d S$

- la concentration moyenne $C_{0}(x, t)=\frac{1}{S} \int_{S} c(\vec{r}, x, t) d S$ 
Nous appellerons $D$ le coefficient de diffusion moléculaire qui règlera les échanges par diffusion (non convectifs) à travers les surfaces du volume de contrôle.

Le bilan de matière dans ce volume de contrôle s'écrira :

$$
\begin{aligned}
\int_{x}^{x+\delta x} d x \int_{S} \frac{\partial c}{\partial t} d S+ & \int_{S(x+\delta x)} u c d S-\int_{S(x)} u c d S- \\
& \int_{S(x+\delta x)} D \frac{\partial c}{\partial x} d S+\int_{S(x)} D \frac{\partial c}{\partial x} d S=0
\end{aligned}
$$

Nous supposons ici que la paroi latérale $S_{L}$ de la veine liquide ne transfère pas de matière.

Si l'on fait tendre $\Delta x$ vers zéro, ce bilan prend la forme :

$$
\int_{S} \frac{\partial c}{\partial t} d S+\frac{\partial}{\partial x}\left(\int_{S} u c d S\right)=\frac{\partial}{\partial x}\left(\int_{S} D \frac{\partial c}{\partial x} d S\right)
$$

Le problème fondamental consiste à écrire cette équation en n'utilisant que la vitesse débitante $U$ pour déterminer la concentration moyenne $C_{0}$.

Considérons d'abord le cas simple des écoulements dans les conduites ou les canaux en régime uniforme pour que $S(x, t)=$ constante (alors $U(t)$ est également indépendant de $x$ ).

Alors seulement les premier et dernier termes n'offrent pas de difficultés :

$$
\begin{aligned}
\int_{S} \frac{\partial c}{\partial t} d S & =\frac{\partial}{\partial t} \int_{S} c d S=S \frac{\partial C_{0}}{\partial t} \\
\frac{\partial}{\partial x} \int_{S} D \frac{\partial c}{\partial x} d S & =D \frac{\partial^{2}}{\partial x^{2}} \int_{s} c d S=S D \frac{\partial^{2} C_{0}}{\partial x^{2}}
\end{aligned}
$$

Mais comme pour l'établissement des équations de Reynolds :

$$
\frac{\partial}{\partial x} \int_{S} \text { uc } d S \neq S \frac{\partial U C_{0}}{\partial x}
$$

On peut, ici également, poser :

$$
\left\{\begin{array}{l}
u=U+U^{\prime}(x, \vec{r}, t) \\
c=C_{0}(x, t)+c^{\prime}(x, \vec{r}, t)
\end{array}\right.
$$

$U^{\prime}$ et $c^{\prime}$ sont tels que :

$$
\int_{s} U^{\prime} d s=\int_{S} c^{\prime} d S=0
$$

Alors :

$$
\frac{\partial}{\partial x} \int_{S} u c d S=S U \frac{\partial C_{0}}{\partial x}+\frac{\partial}{\partial x}\left(\int_{S} U^{\prime} c^{\prime} d S\right)
$$

Comme dans les équations de Reynolds, le dernier terme représente une moyenne, mais c'est une moyenne spatiale et non plus temporelle ou statistique.

Finalement, dans le cas des écoulements filaires uniformes où nous nous sommes mis pour simplifier, l'évolution de la concentration moyenne $C_{0}$ obéit à l'équation :

$$
\frac{\partial C_{0}}{\partial t}+U \frac{\partial C_{0}}{\partial x}=D \frac{\partial^{2} C_{0}}{\partial x^{2}}-\frac{\partial}{\partial x}\left(\frac{1}{S} \int_{S} U^{\prime} c^{\prime} d S\right)
$$

Cette équation appelle de nombreux commentaires.

Le premier membre représente la convection de la concentration moyenne par la vitesse débitante.

Le premier terme du second membre provient de la diffusion moléculaire dans l'axe de l'écoulement. Il est en général négligeable devant le second terme qui traduit la «convection différentielle», c'est-à-dire la convection supplémentaire à celle représentée par la vitesse débitante du premier membre qui ne transporte d'ailleurs que la concentration moyenne.

Tout notre effort va porter sur ce terme de convection différentielle. Il faut d'abord remarquer que s'il représente une moyenne, sur la section, du produit $U^{\prime} c^{\prime}$ des écarts de la vitesse et de la concentration par rapport à leur valeur moyenne respective, ces écarts sont beaucoup plus importants que les fluctuations turbulentes qui sont considérées dans les corrélations turbulentes. Cette convection différentielle dans les écoulements filaires masque donc non seulement la diffusion moléculaires longitudinale, mais aussi la diffusion turbulente longitudinale.

Mais il faudra bien se garder de penser que ces actions diffusives sont à négliger. Nous verrons, au contraire, qu'elles sont primordiales et constituent l'une des charnières déterminantes du mécanisme de transfert des écoulements filaires. En effet, c'est dans le sens transversal et non longitudinal que l'action diffusive est tout à fait essentielle, parce qu'elle va déterminer la répartition de $\mathrm{c}^{\prime}$ dans la section.

Nous devons à l'intuition tout à fait géniale de Taylor d'avoir, le premier, décortiqué ce mécanisme subtil. Il le démonta dans une série d'expériences qu'il présenta en 1953. Pour que sa démonstration fût tout à fait convaincante, il la fit en écoulement laminaire pour ôter tout élément aléatoire qu'aurait pu apporter la turbulence.

Taylor travailla avec des tubes de 0,5 et $1 \mathrm{~mm}$ de diamètre, c'est-à-dire qu'il n'était pas question pour lui d'aller mesurer les répartitions dans les sections et que seules les valeurs moyennes lui étaient accessibles. Par contre, il avait un avantage considérable, c'est qu'il connaissait, sans avoir à la mesurer, la répartition des vitesses. Utilisant une solution de permanganate de potassium $\left(\mathrm{MnO}_{4} \mathrm{~K}\right)$, il déterminait la concentration par colorimétrie, en comparant la couleur dans les différentes sections à celle de tubes témoins à concentration connue. Il interrompait l'écoulement de temps en temps pour faire ses relevés colorimétriques, ces interruptions lui permettant de figer l'écoulement à l'instant qu'il désirait. Il avait au préalable vérifié qu'une distribution longitudinale donnée pouvait rester immuable pendant très longtemps lorsque le fluide était au repos. Cette remarque confirmait d'ail- 
leurs que la diffusion moléculaire longitudinale était tout à fait négligeable.

Le résultat essentiel, et à première vue paradoxal, de ses expériences est que, lorsque l'action diffusive (nécessairement moléculaire dans ses expériences) est négligeable, la dispersion longitudinale de la solution est toujours plus importante que lorsque le phénomène de diffusion existe.

Ainsi, si l'on coince, à l'état initial, un disque de colorant entre des eaux claires, on obtient, après avoir fait couler à la vitesse moyenne $U$ pendant le temps $t$, les résultats suivants :

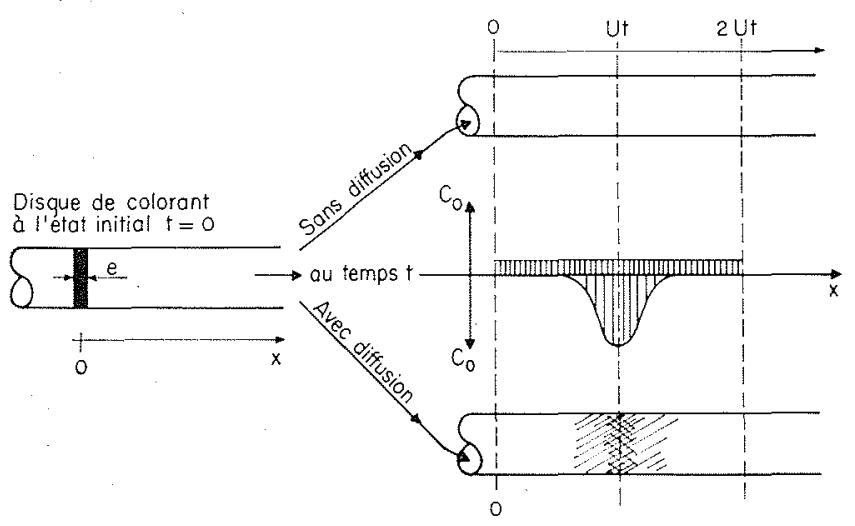

La répartition longitudinale uniforme de la concentration moyenne $C_{0}$ sur une longueur de tuyau égale à $2 U t$, dans le cas où il n'y a pas de diffusion, est très facile à expliquer à partir du profil parabolique des vitesses en écoulement laminaire. On sait, en effet, que ce profil a une vitesse maximum au centre égale au double de la vitesse moyenne.

Mais, dans ce cas, la représentation globale de la convection différentielle au second membre de l'équation d'évolution de $C_{0}$ est compliquée, et ne présenterait d'ailleurs aucun intérêt pratique, car dans la plupart des écoulements que l'on rencontre, il y a de la diffusion et non seulement moléculaire mais même turbulente.

Par contre, ce qui se passe dans le cas où il y a de la diffusion est beaucoup plus intéressant, et nous allons nous attacher à l'expliquer dans ce qui suit. Il est évident que l'allure des résultats expérimentaux nous pousse à chercher à représenter la «convection différentielle»par une diffusion globale de la concentration moyenne $C_{0}$ :

$$
-\frac{1}{S} \int_{S} U^{\prime} c^{\prime} d S=K \frac{\partial C_{0}}{\partial x} ?
$$

Nous allons établir ce résultat et en donnerons également l'explication physique.

\section{Transfert longitudinal moyen par la convection différentielle en présence d'une diffusion transversale}

Le rôle de la diffusion sera, à chaque instant, d'uniformiser la répartition des concentrations dans toutes les sections, en répartissant au hasard le colorant sur les différents convecteurs.

Remarquons également que la diffusion agira plus ou moins, selon que le coefficient de diffusion $D$ sera grand ou petit, ou bien, selon qu'on lui laissera ou non le temps d'agir. C'est d'ailleurs cette seconde interprétation qu'avait choisie Taylor : quand il voulait avoir des résultats sans diffusion, il faisait des expériences dans des tubes de $1 \mathrm{~mm}$ de diamètre pendant des durées de l'ordre de la seconde, alors que, lorsqu'il voulait faire agir la diffusion, il remplaçait ces tubes par des tubes de $0,5 \mathrm{~mm}$ de diamètre et réalisait des écoulements qui duraient des heures.

Nous nous restreindrons aux cas des écoulements filaires uniformes, mais pas nécessairement permanents $[U(t)]$. Nous appellerons :

$a$ : une dimension transversale caractéristique des sections droites $S$ dont le contour sera appelé $\Gamma$;

$L$ : une distance longitudinale caractéristique;

$d$ : le coefficient de diffusion transversale qui pourra aussi être turbulente ( $d$ serait alors une fonction du point dans la section $S$ );

$c$ : la concentration variable dans la section $S$ et le long de l'écoulement;

$u$ : la vitesse longitudinale fonction du point dans $S$;

$U$ : la vitesse moyenne ou débitante :

$$
U=\frac{1}{S} \int_{S} u d S=U_{0} f(t)
$$

$u^{\prime}$ : l'écart à la vitesse moyenne, normé par cette dernière : $u=U_{0}\left[f(t)+u^{\prime}\right]$ avec, par conséquent :

$$
\int_{s} u^{\prime} d S=0
$$

$x$ : désignera l'abscisse longitudinale sur l'axe de l'écoulement filaire et

$\vec{r}$ : repèrera les points dans la section $S$.

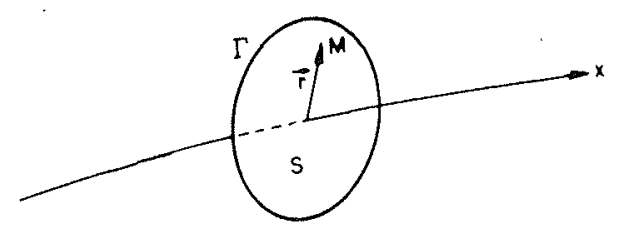

Nous caractériserons le coefficient de diffusion $d(\vec{r})$ par son maximum $D$ par exemple :

$$
D=\underset{S}{\operatorname{Max}} d(\vec{r})
$$


et nous normerons $d$ par $D$ :

$$
d(\vec{r})=D d^{\prime}(\vec{r})
$$

Nous allons, à présent, énoncer les hypothèses que nous avons précédemment discutées et établies :

- l'écoulement est uniquement longitudinal (filaire, donc sans composante de vitesse dans $S$ );

- par contre, la diffusion est uniquement transversale dans $S$, la diffusion longitudinale étant négligeable devant la convection.

- l'écoulement dure assez longtemps pour que la diffusion puisse se développer pleinement :

Or le temps de convection se mesure par : $L / U_{0}=T_{c v}$ et celui de la diffusion transversale par : $a^{2} / D=T_{d i f}$

Donc :

$$
\nu=\mathrm{T}_{a i f} / T_{c v}=a^{2} U_{0} / L D \ll 1
$$

Pour compléter ces notations, faisons remarquer que, dans ce qui suit, tous les opérateurs seront dans $S$, sauf évidemment ceux qui seront explicitement écrits dans $t$ oul $x$.

Ces notations et hypothèses étant précisées, l'équation du bilan local de matières dissoutes s'écrit :

$\frac{\partial c}{\partial t}+u \frac{\partial c}{\partial x}=\operatorname{div}(d \overrightarrow{\operatorname{grad}} c)=\frac{\partial c}{\partial t}+\left(U_{6} f+U_{0} u^{\prime}\right) \frac{\partial c}{\partial x}$

Nous l'écrirons en axes liés à l'écoulement moyen de vitesse $U$, en posant:

$$
\begin{gathered}
x=X+U_{0} f(t) \quad \text { et } \\
\frac{D}{D t}=\frac{\partial}{\partial t}+U_{0} f \frac{\partial}{\partial x} \\
\frac{D c}{D t}+U_{0} u^{\prime} \frac{\partial c}{\partial X}=D \operatorname{div}\left(d^{\prime} \operatorname{grad} c\right)
\end{gathered}
$$

Nous complèterons cette équation indéfinie en précisant les conditions aux limites sur $\Gamma$. Nous supposerons les parois $\Gamma$ «adiabatiques », c'est-à-dire :

$$
\vec{n}_{\Gamma} \cdot \overrightarrow{\operatorname{grad}} c=0
$$

\section{Analyse dimensionnelle du problème}

Nous allons plus exactement procéder à une analyse inspectionnelle.

Tout d'abord, les équations étant homogènes en $c$, il est inutile de normer $c$ qui est d'ailleurs adimensionnel. Toute l'analyse dimensionnelle portera sur l'opérateur différentiel où nous avons explicitement séparé les opérateurs longitudinaux et transversaux :

$$
\left(\frac{D}{D t} \cdot+U_{0} u^{\prime} \frac{\partial}{\partial X} \cdot-D \text { div } d^{\prime} \overrightarrow{\text { grad }} \cdot\right) c=0
$$

Nous sommes conduits à rapporter $X$ à $L$ et $\vec{r}$ à $a$ :

$$
\begin{aligned}
& X=L X^{\prime} \\
& \vec{r}=a \vec{r}^{\prime}
\end{aligned}
$$

et alors :

$$
\begin{gathered}
\frac{\partial}{\partial X}=-\frac{1}{L} \frac{\partial}{\partial X^{\prime}} \\
\operatorname{div}\left(d^{\prime} \text { grad }\right)=\left(1 / a^{2}\right) d i v^{\prime}\left(\mathrm{d}^{\prime} \mathrm{gra}^{\prime}\right)
\end{gathered}
$$

Pour abréger l'écriture, nous noterons

$$
d i v^{\prime}\left(d^{\prime} \operatorname{grad}^{\prime}\right)=\Delta_{d^{\prime}}^{\prime}
$$

En variables spatiales réduites, l'opérateur différentiel de l'équation indéfinie s'écrit :

$$
\begin{aligned}
\frac{D}{D t}+U_{0} u^{\prime} \frac{\partial}{\partial X} & -D \Delta_{a^{\prime}}^{\prime} \\
& =\frac{D}{a^{2}}\left[\frac{a^{2}}{D} \frac{D}{D t}+\frac{U_{0} a^{2}}{D L} u^{\prime} \frac{\partial}{\partial X^{\prime}}-\Delta_{a^{\prime}}^{\prime}\right]
\end{aligned}
$$

Pour le choix du temps de référence, cette expression nous inciterait à placer $t$ dans l'échelle des temps de diffusion $T_{a i f}=a^{2} / D$. Mais on sait que l'opérateur $D / D t$ (qui est une dérivation particulaire) représente la convection moyenne et la physique nous incite plutôt à le mettre à l'échelle des temps de convection $T_{c v}=L / U_{0}$ que nous avons supposée beaucoup plus grande que celle de diffu$\operatorname{sion}\left(v=T_{\text {dif }} / T_{c v} \ll 1\right)$.

Mais nous allons aller plus loin et exagérer cette distorsion des échelles longitudinales et transversales, en rapportant le temps non pas à $T_{c v}$, mais à $T_{c v} / v=T^{2}{ }_{c v} / T_{d i f}$.

$$
t=T^{2}{ }_{c v} t^{\prime} / T_{d i f}=\left(D L^{2} / a^{2} U_{0}^{2}\right) t^{\prime}
$$

Dans ces conditions, l'opérateur s'écrit sous forme entièrement adimensionnelle :

$$
\begin{aligned}
\frac{D}{D t}+U_{0} u^{\prime} \frac{\partial}{\partial X} & -D \Delta_{a^{\prime}} \\
& =\frac{D}{a^{2}}\left[-\Delta_{a^{\prime}}^{\prime}+v u^{\prime} \frac{\partial}{\partial X^{\prime}}+v^{2} \frac{D}{D t^{\prime}}\right]
\end{aligned}
$$

Nous remarquons que le choix d'une norme en temps égale à $T_{c v} / v$ a permis d'ordonner l'opérateur en puissance croissante de $\nu$, alors que le choix de $T_{d i f}$ ou de $T_{c v}$ eût regroupé l'opérateur $D / D t$, avec soit $\Delta_{d^{\prime}}^{\prime}$, soit $u^{\prime}\left(\partial / \partial X^{\prime}\right)$.

\section{Recherche de la solution}

Cette forme de l'équation indéfinie nous incite à rechercher la solution approchée de $c$ en série de puissances entières du petit paramètre $\nu$ :

$$
c=C_{0}+v^{\prime} c_{1}+v^{2} c_{2}+\ldots \ldots
$$

En se bornant aux termes du second ordre, l'équation indéfinie s'écrit :

$$
\begin{aligned}
{\left[\Delta_{a^{\prime}}^{\prime} C_{0}\right]+v\left[\Delta_{d^{\prime}}^{\prime} c_{1}-u^{\prime} \frac{\partial C_{0}}{\partial X^{\prime}}\right] } & \\
+ & +v^{2}\left[\Delta_{l^{\prime}}^{\prime} c_{2}-u^{\prime} \frac{\partial c_{1}}{\partial X^{\prime}}-\frac{D C_{0}}{D t^{\prime}}\right]+\ldots . .=0
\end{aligned}
$$


avec les conditions aux limites :

$$
\vec{n}_{\Gamma^{\prime}}^{\prime} \cdot\left[\overrightarrow{g r a d}^{\prime} C_{n}+v \overrightarrow{\operatorname{grad}^{\prime}} c_{1}+y^{2} \operatorname{grad}^{\prime} c_{2}+\ldots . .\right]=0
$$

- à l'ordre 0 en $v$, on est conduit à résoudre :

$$
\left\{\begin{aligned}
\operatorname{div}^{\prime}\left(d^{\prime} \operatorname{grad}^{\prime} C_{0}\right) & =0 \text { dans } \mathrm{S}^{\prime}, \text { avec } \\
\partial C_{0} / \partial n^{\prime} & =0 \text { sur } \Gamma^{\prime}
\end{aligned}\right.
$$

dont la solution est une constante par rapport à $\vec{r}^{\prime}$, c'est-àdire une fonction uniquement de $X^{\prime}$ et $t^{\prime}$ :

$$
C_{0}=C_{0}\left(X^{\prime}, t^{\prime}\right)
$$

- à l'ordre 1 en $v$, il faut déterminer $c_{1}$ tel que :

$$
\left\{\begin{aligned}
d i v^{\prime}\left(d^{\prime} \overrightarrow{\operatorname{grad}} c_{1}^{\prime}\right) & =u^{\prime}\left(\partial C_{0} / \partial \mathrm{X}^{\prime}\right) \text { dans } \mathrm{S}^{\prime}, \text { avec } \\
\partial c_{1} / \partial n^{\prime} & =0 \text { sur } \Gamma^{\prime}
\end{aligned}\right.
$$

Dans ces équations en $\vec{r}, \partial C_{0} / \partial X^{\prime}$ est un paramètre.

Il faut remarquer que les conditions aux limites sont du type de Neuman et homogènes, et que ceci impose une condition sur le second membre de l'équation indéfinie qui représente une répartition de sources. En effet, intégrons cette équation dans $S^{\prime}$ :

$$
\begin{aligned}
\frac{\partial c_{0}}{\partial X^{\prime}} \int_{S^{\prime}} u^{\prime} d s & =\int_{S^{\prime}} d i v^{\prime}\left(d^{\prime} \operatorname{grad}^{\prime} c_{1}\right) d s^{\prime} \\
& =\int_{\Gamma^{\prime}} \vec{n}^{\prime} d^{\prime} \operatorname{grad}^{\prime} c_{1}=0
\end{aligned}
$$

Or il se trouve que $u^{\prime}$ vérifie bien cette condition par définition.

La présence de sources nous conduit à faire appel à la fonction de Green $G$ du problème. Par définition $G\left(\vec{r}^{\prime}, \vec{r}_{0}^{\prime}\right)$ est solution de :

$$
\left\{\begin{aligned}
d i v^{\prime}\left(d^{\prime} \operatorname{grad}^{\prime} G\right) & =\delta\left(\vec{r}^{\prime}-\vec{r}_{0}^{\prime}\right)-1 \text { dans } S^{\prime}, \text { avec } \\
\partial G / \partial n^{\prime} & =0 \text { sur } \Gamma^{\prime}
\end{aligned}\right.
$$

où $\delta\left(\vec{r}^{\prime}-\vec{r}_{0}^{\prime}\right)$ est une masse de Dirac placée en $\vec{r}_{0}$.

(Sans qu'il soit nécessaire de rentrer dans le détail ici, on peut se contenter de chercher une solution de l'équation indéfinie qui n'aurait au second membre que la masse de Dirac, compte tenu du fait que la répartition des sources $u^{\prime}$ est à valeur moyenne nulle sur $S^{\prime}$ ).

La solution $c_{1}$ s'obtient alors commodément par :

$$
c_{1}\left(\vec{r}^{\prime} ; X^{\prime} t^{\prime}\right)=\frac{\partial C_{0}}{\partial X^{\prime}} \int_{s^{\prime}} G\left(\vec{r}^{\prime}, \vec{r}_{0}^{\prime}\right) u^{\prime}\left(\vec{r}_{0}^{\prime}, t^{\prime}\right) d s^{\prime}\left(\vec{r}_{0}^{\prime}\right)
$$

Nous appellerons $c_{1}^{\prime}\left(\vec{r}^{\prime}, t^{\prime}\right)$ la fonctionnelle définie par l'intégrale :

$$
c_{1}^{\prime}\left(\vec{r}^{\prime} ; t^{\prime}\right)=\int_{S^{\prime}} G\left(\vec{r}^{\prime}, \vec{r}_{0}^{\prime}\right) u^{\prime}\left(\vec{r}_{0}^{\prime}, t^{\prime}\right) d s^{\prime}\left(\vec{r}_{0}^{\prime}\right)
$$

pour mettre en évidence la séparation des variables $X^{\prime}$ et $\vec{r}^{\prime}$ dans $c_{1}$ :

$$
c_{1}\left(\vec{r}^{\prime} ; X^{\prime}, t^{\prime}\right)=\left(\partial C_{0} / \partial X^{\prime}\right) c_{1}^{\prime}\left(\vec{r}^{\prime}, t^{\prime}\right)
$$

- Enfin à l'ordre 2 en $v$, il faut ajouter les équations : $\left\{d i v^{\prime}\left(d^{\prime} \operatorname{grad}^{\prime} c_{2}\right)=u^{\prime}\left(\partial c_{1} / \partial X^{\prime}\right)+\left(D C_{0} / D t^{\prime}\right)\right.$ dans $S^{\prime}$ avec $\left\{\partial c_{2} / \partial n^{\prime}=0\right.$ sur $\Gamma^{\prime}$

La séparation des variables dans $c_{1}$ nous conduit à en faire autant pour $c_{2}$ en posant :

$$
c_{2}\left(\vec{r}, X^{\prime}, t^{\prime}\right)=\left(\partial^{2} C_{0} / \partial X^{\prime 2}\right) c_{2}^{\prime}\left(\vec{r}, t^{\prime}\right)
$$

L'équation indéfinie devient alors :

$$
\left(\partial^{2} C_{0} / \partial X^{\prime 2}\right)\left[d i v^{\prime}\left(d^{\prime} \operatorname{grad}^{\prime} c_{2}^{\prime}\right)-u^{\prime} c_{1}^{\prime}\right]=D C_{0} / D t^{\prime}
$$

Elle se sépare elle-même en :

$$
\begin{gathered}
D C_{0} / D t^{\prime}=K^{\prime}\left(\partial^{2} C_{0} / \partial X^{\prime 2}\right) \text { sur } X^{\prime} \quad \text { et } \\
\operatorname{div}^{\prime}\left(d^{\prime} \overrightarrow{g r a d}^{\prime} c_{2}^{\prime}\right)-u^{\prime} c_{1}^{\prime}=K^{\prime} \text { dans } S^{\prime} \text {, avec } \\
\partial c_{2}^{\prime} / \partial n^{\prime}=0 \text { sur } \Gamma^{\prime}
\end{gathered}
$$

On est de nouveau amené à résoudre dans $S^{\prime}$ un problème de Neuman avec des sources réparties dans $S^{\prime}$ et des conditions aux limites homogènes. Ceci implique encore que la somme des sources soit nulle dans $S_{1}^{\prime}$ ce qui impose à la constante de séparation $K^{\prime}$ d'être égale à :

$$
\begin{gathered}
K^{\prime}=-\frac{1}{S^{\prime}} \int_{S^{\prime}} u^{\prime} c_{1}^{\prime} d S^{\prime}=-\frac{1}{S_{\vec{r}^{\prime}}^{\prime} \int_{\epsilon} S^{\prime}} u^{\prime}\left(\vec{r}^{\prime}\right) \\
{\left[\int_{\vec{r}_{0}^{\prime} \in S^{\prime}} G\left(\vec{r}^{\prime}, \vec{r}_{0}^{\prime}\right) u^{\prime}\left(\vec{r}^{\prime}, \vec{r}_{0}^{\prime}\right) d S\left(\vec{r}_{0}^{\prime}\right)\right] d S\left(\vec{r}^{\prime}\right)}
\end{gathered}
$$

Il est bon de remarquer que $K^{\prime}>0$. En effet :

$$
K^{\prime}=-\frac{1}{S^{\prime}} \int_{s^{\prime}} u^{\prime} c^{\prime}{ }_{1} d s^{\prime}
$$

$c_{1}^{\prime}$ étant défini par :

$$
\Delta_{d^{\prime}}^{\prime} c^{\prime}{ }_{1}=u^{\prime}
$$

En multipliant cette équation par $c_{1}^{\prime}$ et en intégrant sur $S^{\prime}$, on trouve :

$$
\begin{aligned}
K^{\prime} & =-\frac{1}{S^{\prime}} \int_{S^{\prime}} c_{1}^{\prime} \operatorname{div}^{\prime}\left(d^{\prime} \overrightarrow{\operatorname{grad}^{\prime}} c_{1}^{\prime}\right) d S^{\prime} \\
& =\frac{1}{S^{\prime}} \int_{S^{\prime}} d^{\prime}\left(\overrightarrow{\operatorname{grad}^{\prime}} c_{1}^{\prime}\right)^{2} d S^{\prime}>0
\end{aligned}
$$

$\operatorname{car} d^{\prime}>0$ 
Il est sans intérêt de chercher à déterminer $c_{2}^{\prime}$, mais il est essentiel de noter qu'au second ordre en $v, C_{0}\left(X^{\prime}, t^{\prime}\right)$ se trouve précisé par l'équation :

$$
\frac{D C_{0}}{D t^{\prime}}=K^{\prime}\left(t^{\prime}\right) \frac{\partial^{2} C_{0}}{\partial X_{2}^{\prime}}
$$

qu'on aurait pu obtenir en intégrant sur $S^{\prime}$ l'équation originelle définissant $c_{2}$.

Ici s'achève donc la recherche de la solution au second ordre en $v$, mais après ces développements mathématiques, il est bon de refaire le point.

\section{Interprétation physique. Mécanisme de la dispersion longitudinale de la concentration moyenne par la diffusion transversale et la convection différentielle}

1) Tout d'abord, il convient d'identifier physiquement $C_{0}$, le premier terme du développement de $c$ tel qu'on l'a trouvé :

$$
\begin{aligned}
c\left(\vec{r}^{\prime}, X^{\prime}, t^{\prime}\right)=C_{0}\left(X^{\prime}, t^{\prime}\right)+v & \frac{\partial C_{0}}{\partial X^{\prime}} c_{1}^{\prime}\left(\vec{r}^{\prime}, t^{\prime}\right) \\
& \quad+\nu^{2} \frac{\partial^{2} \mathrm{C}_{0}}{\partial X^{\prime 2}} c_{2}^{\prime}\left(\vec{r}^{\prime}, t^{\prime}\right)+\ldots . .
\end{aligned}
$$

On peut toujours ajouter une constante aux fonctions $c^{\prime}{ }_{1}$ et $c_{2}^{\prime}$ de façon que leur valeur moyenne sur $S^{\prime}$ soit nulle. On identifie alors $C_{0}$ à la concentration moyenne dans la section $S$ :

$$
\int_{s} c d s=S C_{0}
$$

de sorte que l'écart par rapport à la concentration moyenne $C_{0}$ et que nous avions appelé $c^{\prime}$, vaut :

$$
\begin{aligned}
c-C_{0}=c^{\prime}=v & {\left[c_{1}+v c_{2}+\ldots . .\right] } \\
& =v\left[\frac{\partial C_{0}}{\partial X^{\prime}} c_{1}^{\prime}+v \frac{\partial^{2} C_{0}}{\partial X^{\prime 2}} c_{2}^{\prime}+\ldots .\right]
\end{aligned}
$$

Soit au premier ordre en $v$ :

$$
c^{\prime}=v \frac{\partial C_{0}}{\partial \bar{X}^{\prime}} c_{1}^{\prime}=L v \frac{\partial C_{0}}{\partial x} c_{1}^{\prime}
$$

2) Nous nous étions posé la question fondamentale de savoir si dans l'équation :

$$
\frac{\partial C_{0}}{\partial t}+U \frac{\partial C_{0}}{\partial x}=\frac{\partial}{\partial x}\left(-\frac{1}{S} \int_{s} U^{\prime} c^{\prime} d S\right)
$$

qui représente le bilan de matière dans une tranche infiniment mince, au second membre, nous pouvions écrire :

$$
-\frac{1}{S} \int_{S} U^{\prime} c^{\prime} d S=K \frac{\partial C_{0}}{\partial x} ?
$$

La réponse apportée par les calculs que nous venons d'effectuer est affirmative, dans l'hypothèse essentielle où :

$$
v=T_{a i f} / T_{c v} \ll 1
$$

En effet à une erreur en $v^{2}$ près :

$$
\begin{aligned}
-\frac{1}{S} \int_{S^{\prime}} u^{\prime} c^{\prime} d S & =--\frac{U_{0}}{S^{\prime}} \int_{S^{\prime}} u^{\prime} L \frac{\partial C_{0}}{\partial x} c_{1}^{\prime} d S^{\prime} \\
& =U_{0} L v\left(-\frac{1}{S^{\prime}} \int_{S^{\prime}} u^{\prime} c_{1}^{\prime} d S^{\prime}\right) \frac{\partial C_{0}}{\partial x}
\end{aligned}
$$

soit :

$$
\begin{gathered}
-\frac{1}{S} \int_{s} U^{\prime} c^{\prime} d S=K \frac{\partial C_{0}}{\partial x} \text { avec } \\
K=K^{\prime} a^{2} U_{0}{ }^{2} / D \quad \text { et } \\
\left.K^{\prime}=-\left.\frac{1}{S} \int_{\overrightarrow{r^{\prime}} \epsilon S} u^{\prime}\left(\vec{r}^{\prime}\right)\right|_{\vec{r}_{0}^{\prime} \epsilon} ^{-} G\left(\vec{r}^{\prime}, \vec{r}^{\prime}\right) u^{\prime}\left(\vec{r}_{0}^{\prime}\right) d S\left(\overrightarrow{\mathrm{r}}_{0}^{\prime}\right)\right] d S\left(\vec{r}^{\prime}\right)
\end{gathered}
$$

Ces formules établissent donc, qu'à condition que

$$
v=T_{a i f} / T_{c v}=a^{2} U_{0} / L D \ll 1,
$$

on peut étudier le transfert global de matière, par un «modèle filaire» qui est une équation de diffusion unidimensionnelle de la concentration moyenne $C_{0}$, à une erreur en $\nu^{3}$ près :

$$
\frac{\partial C_{0}}{\partial t}+U \frac{\partial C_{0}}{\partial x}=K \frac{\partial^{2} C_{0}}{\partial x^{2}}
$$

3) Nous en arrivons, enfin, à l'explication physique de ce résultat. Nous l'obtiendrons par une interprétation physique des calculs que nous avons effectués. Nous retrouverons d'ailleurs l'explication donnée par Taylor. Rappelons que le choix d'un temps de référence égal à $T_{c v}{ }^{2} / T_{a i f}$ a permis d'écrire l'équation du bilan ponctuel de matière sous une forme ordonnée en puissance croissante de $v$.

En résolvant cette équation à l'ordre zéro, nous n'avons trouvé qu'une action de la diffusion transversale imposant une concentration uniforme $C_{0}$ dans la section. Les échelles respectives des temps de diffusion et de convection font que la diffusion atteint son équilibre.

A l'ordre 1 , il apparaît que cette concentration uniforme $C_{0}$ sera perturbée par la répartition de source $u^{\prime}\left(\partial C_{0} / \partial X^{\prime}\right)$. En effet, la convection différentielle commence à jouer, et, dans chaque section, apporte, par le champ de vitesse $u^{\prime}$, des concentrations provenant des sections adjacentes. Ces concentrations venues des sections voisines constituent des sources qui vont immédiatement diffuser modifiant la concentration $C_{0}$ d'une quantité égale à $v c_{1}$. Mais à l'ordre 1 où nous nous trouvons, il suffit de ne faire venir du voisinage que la concentration moyenne $C_{0}$, la modulation de l'intensité des sources provenant surtout de la répartition de $u^{\prime}$ qui amènera dans la section, des concentrations moyennes $C_{0}$ venant de plus ou moins loin.

$\mathrm{Ce}$ ne sera qu'au second ordre en $v$ que l'on tiendra compte de la convection différentielle par $u^{\prime}$ des concentrations $v c_{1}$ des sections voisines. Et c'est seulement à cet ordre qu'apparaîtra l'effet de la convection moyenne $U$ qui n'agit d'ailleurs encore que sur les concentrations moyennes $C_{0}$. Enfin, on trouvera que la convection différentielle de $v c_{1}$, compte tenu de la distribution de $c_{1}$ obtenue à l'ordre précédent, est équivalente à une diffusion longitudinale de la concentration moyenne $C_{0}$. 
Nous résumerons ce mécanisme par le schéma suivant :

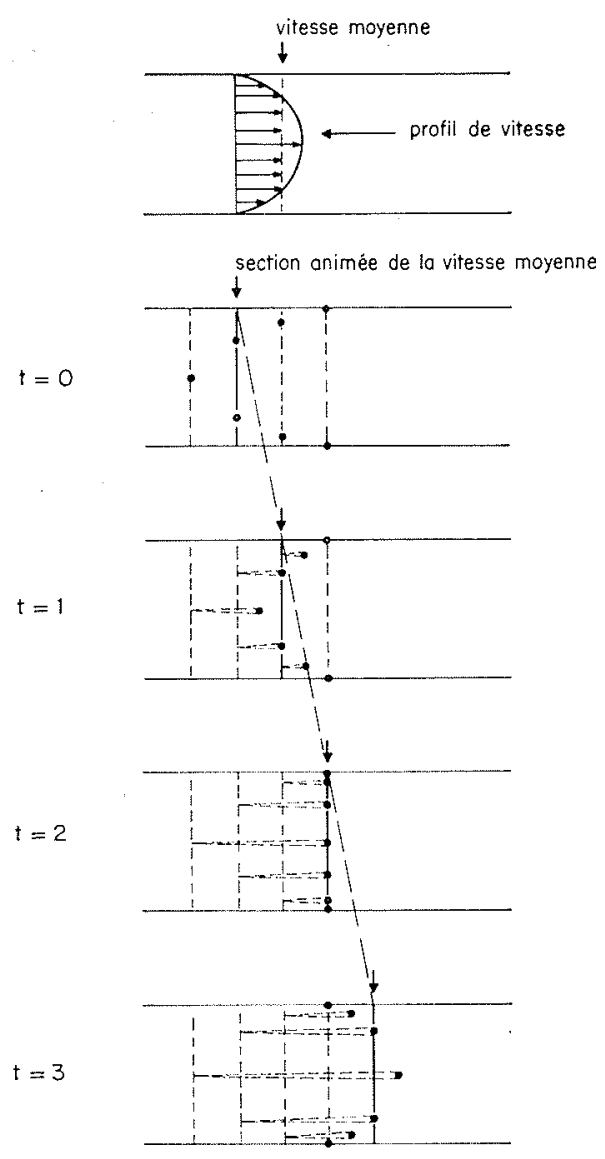

\section{Applications}

\section{Détermination pratique du coefficient de dispersion globale longitudinale}

C'est ainsi que nous nommerons $K$ dans l'équation filaire de la concentration moyenne $C_{0}$.

En pratique, sa détermination se fait en deux temps, après avoir fait le choix des grandeurs de références:

a pour la dimension transversale;

$U_{0}$ pour la vitesse;

$D$ pour le coefficient de diffusion transversale.

Par rapport à ces grandeurs de référence, la répartition de vitesse relative à la vitesse débitante s'écrira $u^{\prime}$ et le coefficient de diffusion $d^{\prime}$.

\section{Première ÉtAPe}

Déterminer $c^{\prime}{ }_{1}$ par :

$$
\left\{\begin{aligned}
\operatorname{div}^{\prime}\left(d^{\prime} \operatorname{grad}^{\prime} c_{1}^{\prime}\right) & =u^{\prime} \text { dans } S^{\prime} \\
\vec{n}^{\prime} \cdot \operatorname{grad}^{\prime} c_{1}^{\prime} & =0 \text { sur } \Gamma^{\prime}
\end{aligned}\right.
$$

Nous donnerons plus loin la solution de cette équation dans deux cas.

DEUXIÈME ÉTAPE

Calculer :

$$
K^{\prime}=-\frac{1}{S^{\prime}} \int_{s^{\prime}} u^{\prime} c_{1}^{\prime} d s^{\prime}
$$

et finalement :

$$
K=K^{\prime} a^{2} U_{0}^{2} / D \quad \text { ou } \quad K=K^{\prime} P_{e}^{2} D
$$

$P_{c}$ étant le nombre de Péclet $a U_{0} / D$.

\section{REMARQUE :}

Dans le cas où l'on connaîtrait la fonction de Green $G$ du problème défini dans la première étape, on peut directement calculer $K^{\prime}$ par :

$$
\begin{aligned}
\mathrm{K}^{\prime}=-\frac{1}{S^{\prime}} \int_{S^{\prime}} u^{\prime}\left(\vec{r}^{\prime}\right) \\
\\
{\left[\int_{S^{\prime}} G\left(\vec{r}^{\prime}, \vec{r}_{0}^{\prime}\right) u^{\prime}\left(\vec{r}_{0}^{\prime}\right) d s^{\prime}\left(\vec{r}_{0}^{\prime}\right)\right] d s^{\prime}\left(\vec{r}^{\prime}\right) }
\end{aligned}
$$

\section{Cas particuliers des conduites circulaires ou des canaux très larges}

Dans ces deux cas, le problème à résoudre en première étape se ramène à une dimension et l'intégration se fait aisément. En particulier, la fonction de Green s'écrit alors :

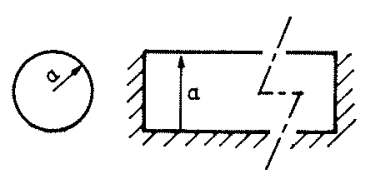

$$
G\left(r^{\prime}, r_{0}{ }^{\prime}\right)=\left\{\begin{array}{l}
0 \text { si } r^{\prime}<r_{0}^{\prime} \\
\int_{r_{0}^{\prime}}^{r^{\prime}} \frac{d \zeta}{(2 \pi \zeta)^{n} d^{\prime}(\zeta)} \text { si } r^{\prime}>r_{0}^{\prime} \\
\text { avec } n=\left\{\begin{array}{l}
0 \text { pour le canal } \\
1 \text { pour la conduite }
\end{array}\right.
\end{array}\right.
$$

Et l'on trouve finalement pour $K^{\prime}$ :

$$
\begin{aligned}
K^{\prime}=- & \int_{0}^{1}\left(2 r^{\prime}\right)^{n} u^{\prime}\left(r^{\prime}\right) d r^{\prime} \int_{0}^{r^{\prime}} \frac{d r_{0}{ }^{\prime}}{\left(r_{0}{ }^{\prime n} d^{\prime}\left(r_{0}{ }^{\prime}\right)\right.} \\
& \int_{0}^{r_{0}{ }^{\prime}}\left(r_{1}{ }^{\prime}\right)^{n} u^{\prime}\left(r_{1}{ }^{\prime}\right) d r_{1}^{\prime}
\end{aligned}
$$

avec les valeurs de $\mathrm{n}$ précédemment définies.

Cette formule avait été établie par Elder en appliquant le raisonnement de Taylor, alors que Taylor qui étudia 
lui-même, le premier, la dispersion dans les conduites ne l'a jamais explicitement écrite.

\section{Résultats numériques}

Dispersion EN CONDUITE CIRCULAIRE (Taylor)

- écoulement laminaire :

$\left\{\begin{array}{l}U_{0}=\text { vitesse débitante; } \\ D=\text { coefficient de diffusion moléculaire; }\end{array} \Rightarrow K^{\prime}=1 / 48\right.$

- écoulement turbulent :

$U_{0}=\sqrt{\tau_{0}} / \rho=$ vitesse de frottement $u_{*} ;$

$D=a u_{*}$ et un profil logarithmique de vitesse;

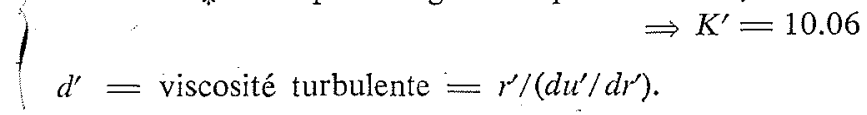

Remarquer que $P_{e}=1$ et $K=K^{\prime} a u_{*}$. Ceci est valable pour les résultats turbulents en canal qui vont suivre.
Dispersion en CANAL RECTANGULAIRE TRÈs LARGe :

- écoulement laminaire :

$U_{0}=$ vitesse débitante;

$D=$ coefficient de diffusion moléculaire;

$\Rightarrow K^{\prime}=7 / 48$

- écoulement turbulent :

$\left\{\begin{array}{l}U_{0}=\sqrt{\tau_{8} / \rho}=u_{*} \\ D=a u_{*}\end{array}\right.$

Elder :

$u^{\prime}$ défini par un profil de vitesse logarithmique;

$\Rightarrow K^{\prime}=5.86$

$d^{\prime}=\left(1-\mathrm{r}^{\prime}\right) /\left(d u^{\prime} / d r^{\prime}\right)=$ viscosité turbulente;

Sayre et Chang :

$\left\{\begin{array}{l}u^{\prime}=1 / x\left(-3 \mathrm{r}^{\prime 2}+6 \mathrm{r}^{\prime}-2\right) ; \\ d^{\prime}=x / 6, x \text { constante de Karman }=0.4 .\end{array} \Rightarrow K^{\prime}=6.65\right.$ 